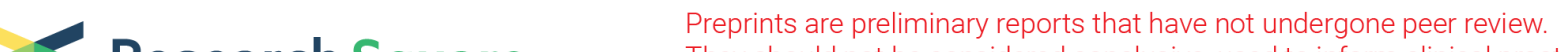 Research Square They should not be considered conclusive, used to inform clinical practice, or referenced by the media as validated information.
}

\section{Molecular Cloning, Bioinformatics Analysis and Overexpression of the Tyrosine Aminotransferase Gene in Rehmannia Glutinosa}

\author{
yanqing zhou ( $\nabla$ yqzhou@htu.cn ) \\ Henan Normal University \\ jialin Zhu \\ Henan Normal University \\ dandan Zhang \\ Henan Normal University \\ mengmeng Guo \\ Henan Normal University \\ huimin Li \\ Henan Normal University \\ hongying Duan \\ Henan Normal University
}

Original Article

Keywords: Rehmannia glutinosa, acteoside, RgTAT, cDNA cloning, gene expression

Posted Date: June 9th, 2021

DOI: https://doi.org/10.21203/rs.3.rs-586755/v1

License: () (1) This work is licensed under a Creative Commons Attribution 4.0 International License.

Read Full License 


\section{Abstract}

Rehmannia glutinosa is an important medicinal plant producing many bioactive compounds such as catalpol, acteoside and so on. Tyrosine aminotransferase (TAT) is the first key enzyme that catalyzes the reversible interconversion of tyrosine and 4-hydroxyphenylpyruvate in the tyrosine-derived branch pathway of acteoside biosynthesis. To confirm its role for acteoside accumulation, we isolated a fulllength cDNA from Rehmannia glutinosa Libosch. Sequence analysis indicated that it contained a 1266 bp open reading frame, encoding a TAT of 421 amino acid residues. Multiple sequence alignment revealed that the homology of RgTAT amino acid sequence to that of Sesamum indicum(XP_011100354.1) was the highest (89.94\%). Evolutionary tree showed that Sesamum indicum TAT and RgTAT were grouped together. Quantitative real-time PCR analysis indicated that the expression of $R g T A T$ in leaves was much higher than in roots and stems,and that the expression levels of $R g T A T$ in the tuberous roots, stems and leaves of high-acteoside cultivar BJ-3 were higher than in that of lowacteoside cultivar Wen85-5. A plant expression vector was constructed containing the RgTAT and hygromycin resistance gene $(H y g)$. Transgenic Rehmannia glutinosa Libosch overexpressing $R g T A T$ was obtained via an Agrobacterium tumefaciens-mediated transformation system, in which Hyg expression was confirmed by PCR. RgTAT expression in transgenic plantlets measured by real-time quantitative PCR was $7.72 \pm 0.17$ times greater than its expression in the untransformed plantlets. Moreover, HPLC analysis indicated that enhanced RgTAT expression corresponded to significantly increased acteoside for transgenic plantlets. Our results elucidate the role of $R g T A T$ in the acteoside biosynthesis in Rehmannia glutinosa.

\section{Introduction}

Rehmannia glutinosa Libosch is a valuable medicinal and industrial crop from the Scrophulariaceae family, and widely used in Traditional Chinese medicine, pickled vegetables, beverages, liquors, and horticulture. In traditional medicine, it is prescribed to treat menopause, impotence, alopecia, and other hormone deficiencies. It is endemic to China, Japan, and Korea (Li et al., 2018). In China, it is distributed in Henan, Shandong, Shanxi, Shan'xi and other provinces, but better Rehmanniae Radix is from Jiaozuo, Henan, P.R.China. called Huaidihuan, which has higher bioactive components such as catalpol and acteoside, and lower clinical dosage than that from other places.

Acteoside is a phenylethanoid glycoside, and possesses some bioactivities such as anti-inflammatory, anti-bacterial, antioxidant, anti-fungal, anti-tumor, photo-protective, liver-protective, neuro-protective properties (Alipieva et al., 2014; Zhou et al., 2016; Li et al., 2018). It was first isolated from mullein, also found in other plant species and produced by in vitro plant culture systems (Alipieva et al., 2014). Now, commercially available verbascoside is isolated and purified from its native plants, for example, Herha Cistanches, but there is lack of its resource in that there are the following: (1) world-wide demand for its native plants as a herbal supplement has caused them to be over-exploited. (2) urbanization reduces the land for growing them. Therefore, it urgently requires modern biotechnologies such as gene engineering 
and metabolic engineering for its biosynthesis like other bioactive natural products (O'Connor 2015). What is more, these biotechnologies need a better understanding of acteoside biosynthesis pathway.

Acteoside is synthesized via a phenylpropanoid branch pathway and a tyrosine-derived branch pathway. Tyrosine aminotransferase (TAT) is the first key enzyme that catalyzes the reversible interconversion of tyrosine and 4-hydroxyphenylpyruvate in the tyrosine-derived branch pathway of acteoside biosynthesis (Zhou et al., 2020). Plant TAT genes have been proposed to be important in response to abiotic stress, including drought and osmotic stresses (Wang et al., 2018) and oxidative stress (Ipson et al., 2019), and to take part in rosmarinic acid synthesis (Lu et al., 2013). In addition, the cloning of TAT genes has been reported from some plant species in NCBI. However, there are few reports on RgTAT cloning and its function in acteoside biosynthesis of Rehmannia glutinosa.

Thus, to validate the role of RgTAT for acteoside biosynthesis, we isolated it from Rehmannia glutinosa, and characterized the phylogenetic relationships among TATs from plants, examined its expression profiles in different tissues, constructed its overexpression vector transformed it into Rehmannia glutinosa via an Agrobacterium-mediated transformation system, and discovered the significant increase of acteoside content in transgenic Rehmannia glutinosa by its overexpression. Our studies will lay the foundation of understanding of the role of $R g T A T$ for acteoside biosynthesis.

\section{Materials And Methods}

\section{Plant material}

The cultivars of Rehmannia glutinosa libosch, such as low verbascoside cultivar and high verbascoside cultivar (Chai et al., 2013), grown in cultivated land in Wen County, Henan, China. Their roots, stems and leaves were used as the experimental materials. The young leaves from plant tissue culture-derived cultivar Wen 85-5 plantlets were used for RgTAT genetic transformation, which were cultured in autoclaved Murashige and Skoog (MS) basal medium.

\section{RT-PCR and RT-qPCR analyses}

Based on known plant TAT enzymes and their EC numbers, their genes were reversely inferred and searched from NCBI. RNA extraction, RT-PCR and RT-qPCR analyses were carried out as stated by Zhou et al.(2016). Primers for these genes were designed by DNAMAN software based on the conserved sequences of their sequences from Erythranthe guttatus (XM_012976257.1), Sesamum indicum (XM_011102052.2), Salvia miltiorrhiza (KF220575.1), Scutellaria baicalensis (KF926690.1) and Vitis vinifera (XM_002276428.3).

\section{RgTAT cloning}

The full-length cDNA was cloned in three successive steps. First, RNA extraction and RT-PCR were carried out as stated by Zhou et al.(2016). Second, based on the cDNA fragment of RgTAT cloned by homology cloning method mentioned above, together with Rehmannia glutinosa SRA database in NCBI, Full-length 
cDNA was cloned by electronic cloning via the searching by tBLASTn and assembling with DNAMAN software of ESTs. Third, according to its coding sequence, a pair of primers were designed (Table 1). Its coding sequence (RgTAT) was amplified by RT-PCR(Zhou et al., 2016). The PCR product was cloned into the pMD19-Tvector (TAKARA, Japan). The pMD19-Tvectors with RgTAT were transformed into Ecoli strain DH5a, followed by PRC test and sequencing. The PCR products were fractionated on agarose gel and purified with OMEGA Gel Extraction Kit (Thermo Fisher Scientific, Japan).

\section{Construction of plant expression vector}

The vector pCAMBIA1300-35S, containing the nptIl (neomycin phosphotransferase) gene coding for kanamycin resistance and Hyg $(R)$ as a plant-selectable markers, was chosen for this experiment. A fragment containing the CDS was amplified with the primer pair RgTAT-F with the Xbal site and RgTAT-R with the Kpnl site (Table 1) under the following PCR conditions: 1 cycle of $94^{\circ} \mathrm{C}$ for $5 \mathrm{~min}, 35$ cycles of $94^{\circ} \mathrm{C}$ for $30 \mathrm{~s}, 55^{\circ} \mathrm{C}$ for $30 \mathrm{~s}$ and $72^{\circ} \mathrm{C}$ for $2 \mathrm{~min}$, and finally 1 cycle at $72^{\circ} \mathrm{C}$ for $10 \mathrm{~min}$. Then the amplified fragment was subcloned into the pCAMBIA1300-35S. Recombinant plasmids (pCAMBIA1300-RgTAT) were identified by restriction analysis of purified plasmid DNA, were sequenced, and then mobilized into the Agrobacterium tumefaciens strain GV3101 using the freeze-thaw method and maintained in yeast extract broth (YEB) medium, supplemented with $50 \mathrm{mg} / \mathrm{L}$ rifampicin (rif) and $50 \mathrm{mg} / \mathrm{L}$ kanamycin (kan), followed by PCR identification.

Table 1 The primers and their sequences

\begin{tabular}{|c|c|c|c|}
\hline Candidate genes & Primers & Primer sequences $\left(5^{\prime}-3^{\prime}\right)$ & Amplicons \\
\hline \multirow[t]{2}{*}{ TAT fragment } & TAT-F & AGCCCGTTTGTTCCGATGG & \multirow[t]{2}{*}{$110 \mathrm{bp}$} \\
\hline & $T A T-\mathrm{R}$ & GTGACAAGCCAGCCAAGCC & \\
\hline \multirow[t]{2}{*}{$\operatorname{RgTAT}$} & RgTAT-F & ATGGAGAACGGAGGGTC & \multirow[b]{2}{*}{$1266 b p$} \\
\hline & RgTAT-R & TCATTGTTTCTTAGCGTGTC & \\
\hline \multirow[t]{2}{*}{ TIP41 } & TIP41-F & TGGCTCAGAGTTGATGGAGTGCT & \multirow[t]{2}{*}{$74 \mathrm{bp}$} \\
\hline & TIP41-R & СTCTCCAGCAGCTTTCTCGGAGA & \\
\hline \multirow[t]{2}{*}{ Hyg } & Hyg-F & ATCGAAATTGCCGTCAACC & \multirow[t]{2}{*}{794 bp } \\
\hline & Hyg-R & ACAGCGTCTCCGACCTGAT & \\
\hline
\end{tabular}

\section{Generation of transgenic plantlets}

The pCAMBIA1300-RgTAT was introduced into Rehmannia glutinosa by Agrobacterium-mediated transformation as stated by Zhou et al (2016). The original vector pCAMBIA1300-35S was also transformed. For transformation, the young explants from 10-day-old Rehmannia g/utinosa plantlets were 
cut into about $0.5 \mathrm{~cm}$ pieces and then precultured, immersed in the Agrobacterium suspension, removed, blotted, and co-cultured on a solid MS medium supplemented with $0.1 \mathrm{mg} / \mathrm{L} \mathrm{NAA}, 2.0 \mathrm{mg} / \mathrm{L} 6-\mathrm{BA}$ and $100 \mu \mathrm{mol} / \mathrm{L}$ AS in dark at $25 \mathrm{C}$ for 2 days. After co-cultivation, the explants were washed once with sterile water and transferred to a solid callus-inducing and shoot-differentiating medium (MS basal medium supplemented with $0.1 \mathrm{mg} / \mathrm{L} \mathrm{NAA}, 2.0 \mathrm{mg} / \mathrm{L} 6-\mathrm{BA}, 100 \mu \mathrm{mol} / \mathrm{LAS}$, Hyg (10mg/L) and cefotaxime $(100 \mu \mathrm{mol} / \mathrm{L})]$, and cultured for $3-4$ weeks under a $16 \mathrm{~h}$ photoperiod at $25^{\circ} \mathrm{C}$ to induce calli or shoot formation. Three weeks, the shoots were transferred onto a root-inducing medium containing $1 \mathrm{mg} / \mathrm{L}$ NAA and $10 \mathrm{mg} / \mathrm{L}$ Hyg for rooting. The regenerated plantlets with well-developed shoots and roots (To) were finally transplanted into pots and then transferred to the greenhouse.

\section{Hyg analysis of transgenic plantlets}

Total genomic DNA was extracted from leaves of Rehmannia glutinosa $\mathrm{T}_{0}$ and non-transgenic Rehmannia glutinosa plantlets. The amplification reaction was carried out with a pair of primers (Table1) as stated by Zhou et al (2017). The amplified products were separated by electrophoresis on a $0.8 \%$ agarose gel and analyzed to rapidly screen putative to on selective media.

\section{Gene expression analysis by real-time quantitative PCR}

Total RNA isolated from $\mathrm{T}_{0}$ transgenic Rehmannia glutinosa lines was treated with RNase-free DNase, whose quality and integrity were confirmed with agarose gel electrophoresis. Real-time quantitative PCR was performed with a pair of primers (Table1) as stated by Zhou et al (2016) using $\mathrm{ddH}_{2} \mathrm{O}$ as negative controls lacking the cDNA template and RgTIP41 as an internal control to normalize expression (Table1), and gene expression was quantified using the $2^{-\Delta \Delta C t}$ method.

\section{Measurement of acteoside content in transgenic Rehmannia glutinosa}

Acteoside was extracted from $\mathrm{T}_{0}$ transgenic Rehmannia glutinosa lines and wild type Rehmannia glutinosa separately. $0.2 \mathrm{~g}$ dried samples were ground into a powder, extracted, whose final extract was used for HPLC analysis as reported by Ouyang (2017).

\section{Statistical analysis}

Statistical analysis was conducted with SPPS software (version 19.0) as stated by Zhou et al.(2016). Analysis of variance (ANOVA) was performed.

\section{Results}

\section{RgTAT cloning}

A pair of primers (RgTAT-F1 and RgTAT-R1) were designed based on the conserved region of the TAT genes from other plant species in NCBI and used to amplify a 110-bp cDNA fragment. As a result, a specific cDNA fragment from Rehmannia glutinosa was obtained and then sequenced; Subsequently, 
based on this fragment, a 1266-bp cDNA (ORF) was cloned by electronic cloning method, and then amplified with a pair of primers (RgTAT-F2 and RgTAT-R2), followed by harvest from the agrose gel (Figure 1), purification and sequencing.

Figure 1 The full-length cDNA (RgTAT)

$\mathrm{M} \otimes T$ Trans $2 \mathrm{~K}^{\circledR}$ DNA marker, 1-2: RgTAT

\section{Homology analysis of RgTAT}

The base sequence of RgTAT and the amino acid sequence of its coding protein were shown in Figure 2. RgTAT was submitted to the GenBank database (accession number: MN648812). A multiple amino acid sequence alignment using a Blast search within the GenBank database in NCBI indicated that RgTAT protein was homologous to the TATs from other plant species, ranging from 59.53\% [Arabidopsis thaliana (NP_198465.3)], to 73.18\% [Theobroma cacao (XP_017971878.1)], to 82.12\% [Scutellaria baicalensis (AIV98133.1)], to 87.76\% [Erythranthe guttatus (XP_012831711.1)] and to 89.94\% [Sesamum indicum (XP_011100354.1)] (Figure 3).

Based on the deduced amino acid sequences of RgTAT and other TAT proteins from other plant species, a phylogenetic tree was constructed to study their evolutionary relationships (Figure 4), in which TAT proteins could be grouped into three groups. Among them, only Group 3 had several sub-branches, in which RgTAT had the closest genetic relationship the TAT from Sesamum indicum.

\section{The spatial and phenotype-dependent expression analyses of RgTAT}

The tuberous root RNAs, stem RNAs and leave RNAs from the low verbascoside Wen85-5 plants and the high verbascoside Beijing No.3 plants (BJ-3) were used for qRT-PCR analysis(Zhou et al., 2016), in which TIP41 was internal reference gene (Table 1). RgTAT was expressed in all the tested tissues from both Wen85-5 plants and BJ-3 plants in varying degrees (Figure 5). Its expression level is the highest in the leaves of both cultivars, while its expression level is the lowest in the stems of both cultivars. Moreover, the expressions of RgTAT in the roots, stems and leaves of BJ-3, were higher than in that of Wen85-5. Among them RgTAT expression levels were extremely significantly in both stem and leaves of both varieties of Rehmannia glutinosa $(\mathrm{P}<0.01)$ (Figure 5).

It is correlated known that TyDC expression positively correlated to verbascoside content of $R$. glutonisa cultivar(Wang et al., 2017; Li e al., 2018). In order to validate the role of RgTAT for Rehmannia glutinosa verbascoside biosynthesis, we used TyDC gene as a positive control to investigate both expression correlation. Our result showed that the expression level of $R g T A T$ was positively correlated to that of $T y D C$ in the tuberous roots of Wen85-5 plants and BJ-3 plants(Figure 6). 


\section{The construction of the recombinant plasmid pCAMBIA1300-35S - RgTAT}

The recombinant vector pCAMBIA2300-35S-RgTAT could be constructed by the cloning of RgTAT into the $\mathrm{Xbal}$ and $\mathrm{Kpn} /$ sites of the vector pCAMBIA2300-35S, in which RgTAT was confirmed by double digestion (Figure 7). Then, this recombinant plasmid was introduced into Agrobacterium tumefaciens strain GV3101 and verified by PCR with a pair of primers (Table 1) (Figure 8). Finally, RgTAT was integrated into the chromosomal DNA of Rehmannia glutinosa with an Agrobacterium-mediated transformation system.

\section{Regeneration of transgenic plants}

Our preliminary experiments for callus induction indicated that $10 \mathrm{mg} / \mathrm{L}$ Hyg could completely inhibit callus induction in uninfected explants of Rehmannia glutinosa, so it was used as a selection pressure in the selection medium. After co-cultivation for 2 days (Figure 9a), Agrobacterium-infected pieces of the leaves were cultured on the callus inducing medium to induce calli formation (Figure 9b). Resistant, bright green calli were transferred to the shoot inducing selection medium (Figure 9c), and about $10 \%$ of the co-cultured explants formed shoots. When about $2.0 \mathrm{~cm}$ high, they were cut out of the calli, followed by their transfer to the root-inducing medium for rooting (Figure 9d). There transgenic plants were called T0 transformants (Figure 9e). Then, they were transplanted into flower pots (Figure 9f).

\section{Molecular verification of transgenic plants}

The genomic DNA was isolated from the leaves of two $\mathrm{T}_{0}$ transgenic Rehmannia glutinosa lines and untransformed controls, and used as templates for PCR. The PCR analyses showed that the expected 750 bp was amplified (Figure10). RgTAT mRNA expression in transgenic plant lines was measured by realtime quantitative PCR. Its levels were $7.72 \pm 0.17$ times in No. $1 \mathrm{~T}_{0}$ transformant, and $7.28 \pm 0.13$ times in No.2 $\mathrm{T}_{0}$ transformant greater than its expression in the untransformed plants.

\section{HPLC-based acteoside content analyses}

Acteoside contents in the leaves of the untransformed Rehmannia glutinosa plantlets (CK) and individual transgenic plantlets were detected by HPLC. Two Hyg-resistant transgenic plants had variable and higher acteoside contents (one $2.89 \pm 0.15 \% \mathrm{DW}$, the other $3.12 \pm 0.16 \% \mathrm{DW}$ ) than CK $(1.96 \pm 0.13 \% \mathrm{DW})$. There was a significant correlation between acteoside content and relative expression level of RgTAT as R2 is equal to $0.83(p<0.05)$.

\section{Discussion}

Acteoside is composed of hydroxytyrosol moiety, which is synthesized from tyrosine through tyramine and dopamine, and caffeic acid moiety, which is produced from phenylalanine via a cinnamate pathway, and rhamnose moiety and glucose moiety (Qi et al., 2013). Its biosynthesis begins with the generation of phenylalanine and tyrosine precursors by the shikimate pathway (Alipieva et al., 2014), which were called phenylpropanoid pathway and tyrosine-derived pathway, respectively. The putative pathway of 
verbascoside biosynthesis was established in olive, and its early steps and its upstream intermediates have been known (Alipieva et al., 2014). Some key enzymes and enzymes-corresponding genes were discovered in Rehmannia glutinosa root by transcriptome sequencing (Zhou et al., 2016; Wang et al., 2017). Phenyl ammonia lyase (PAL), the first key enzyme in the phenylpropanoid pathway, is very important for acteoside biosynthesis, and its gene was overexpressed in Rehmannia glutinosa (Xie et al., 2014). Tyrosine-derived pathway was divided into general and specialized tyrosine metabolism pathways (Xu et al., 2020; Zhou et al., 2020). Tyrosine decarboxylase (TyDC) is a key enzyme in the tyrosine-derived pathway of acteoside biosynthesis pathway, converting tyrosine to tyramine, and its gene, together with PAL, was also overexpressed in transgenic plants including Penstemon barbatus (Can.) Roth (Xie et al., 2019). Both overexpressions could increase acteoside content in both plants (Xie et al., 2019). However, other key acteoside biosynthesis-associated genes are still not overexpressed in this plant until now.

Tyrosine aminotransferase (TAT, EC 2.6.1.5) is the first key enzyme that catalyzes the reversible interconversion of tyrosine and 4-hydroxyphenylpyruvate in the tyrosine-derived pathway for syntheses of important secondary metabolites and compounds. Moreover, many TAT genes have been cloned from different plant species (Ipson et al., 2019; Wang et al., 2018; Xu et al., 2020). Now, there are several reports about TAT overexpressions in potato for cellulose biosynthesis and Perilla frutescens for rosmarinic acid (Li 2007; Lu et al., 2013), but there is not any report about TAT overexpression for acteoside biosynthesis in Rehmannia glutinosa. In Tyrosine-derived pathway of acteoside biosynthesis, TAT converts L-tyrosine to 4-hydroxyphenylpyruvate (4HPP) (Zhou et al., 2020) or (pHPP), followed by its conversion to 4hydroxyphenylacetaldehyde (4-HPAA). Tyrosine is also converted to 4-HPAA through decarboxylationoxidative deamination catalyzed by 4-hydroxyphenylacetaldehyde synthase (4HPAAS, EC 4.1.1.108), 4HPAA is a key intermediate in the biosynthesis of tyrosol-derived specialized metabolites (e.g., salidroside in Rhodiola) (Xu et al., 2020). Moreover, salidroside could be converted to acteoside (Zhou et al. 2020). Therefore, RgTAT was cloned from Rehmannia glutinosa and characterized for the first time. Sequence alignment analysis indicated that RgTAT was homologous to TAT family members in other previously identified plants. Phylogenetic tree analysis revealed that RgTAT was homologous to the TATs from known plants, and the closest to the TAT from Sesamum indicum $\mathrm{L}$.

The genotype-specific expression analysis showed that the expressions of RgTAT gene was greater in the roots, stems and leaves of high acteoside cultivar Beijing No.3 plants than in low verbascoside cultivar Wen85-5, suggesting its decisive roles for acteoside biosynthesis, which is similar to those validated acteoside biosynthesis-associated genes in R. glutinosa (Zhou et al., 2016; Wang et al., 2017); It is known that TyDC possesses the decisive role for acteoside biosynthesis(Zhou et al., 2016; Wang et al., 2017). Therefore, after our genotype-dependent expression analysis, we further studied the correlation of $T y D C$ expression to TAT expression. It was found that both were positive correlation (Fig. 5), suggesting the decisive role of TAT for acteoside biosynthesis.

The Agrobacterium tumefaciens-mediated transformation system has been widely used for engineering many plant species (Liu et al., 2020). In our study, RgTAT was transferred into Rehmannia glutinosa with this transformation system, and its regeneration systems were established. PCR analysis confirmed the 
existence of $H y g$ in the transgenic plantlets. qRT-PCR analysis confirmed RgTAT expression in the transgenic plantlets was higher than in controls.

HPLC analysis revealed that acteoside content 1.5-2.0 fold higher than the control were suggesting $R g T A T$ overexpression in the transgenic plantlets. This result demonstrated the critical role of $R g T A T$ for acteoside biosynthesis in transgenic Rehmannia glutinosa. Our statistics result from RgTAT transgenic Rehmannia glutinosa exhibited a positive correlation between its mRNA levels and acteoside content, suggesting that the increase of $R g T A T$ transcription lead to more acteoside synthesis.

\section{Conclusions}

We successfully cloned, spatially expressed and overexpressed RgTAT from Rehmannia glutinosa, and then established its transgenic plantlets with the increased acteoside. The results indicate the key role of RgTAT for acteoside biosynthesis pathway, and lay a foundation for future regulating RgTAT expression and enhancing acteoside content in Rehmannia glutinosa via transgenic technology and gene editing.

\section{Declarations}

\section{Ethics approval and consent to participate}

Not applicable.

\section{Consent for publication}

Not applicable.

\section{Availability of data and materials}

Not applicable.

\section{Competing interests}

The authors declare that they have no competing interests.

\section{Funding}

This work was supported by National Natural Science Foundation of China (31870312), Natural Science Foundation of Henan province (No. 182300410018) and Key R \& D and promotion projects in Henan Province(No. 212300410020).

\section{Authors' contributions}

YZ put forward the research and revised the manuscript. JZ, DZ, and MG carried out the preparation and treatment of test materials, performed the experiment, analyzed the data, and wrote the manuscript. HD 
and $\mathrm{HL}$ helped $\mathrm{YZ}$ to supervise graduates and designed the experiment. All authors contributed comments to the manuscript. All authors read and approved the final manuscript.

\section{Acknowledgments}

We are grateful to Director Yongkang Liu, Wenxian Institute of Agricultural Sciences for his provision of Rehmannia glutinosa cultivars.

\section{Author details}

College of Life Sciences, Henan Normal University, Xinxiang 453007, HN, P.R.China.

\section{References}

1. Alipieva K, Korkina L, Orhan IE, Georgiev MI (2014) Verbascoside-a review of its occurrence, (bio)synthesis and pharmacological significance. Bio Adv 32(6):1065-1076

2. Chai M, Dong CM, Jiang DH, Yao F, Wang XS (2013) Determination of Catalpol and Verbascoside of Different Cultivars Rehmannia glutinosa Libosch. by High Performance Liquid Chromatography. 28 (05), 690-692 + 771

3. Ipson BR, Green RA, Wilson JT, Watson JN, Faull KF, Fisher AL (2019) Tyrosine aminotransferase is involved in the oxidative stress response by metabolizing meta-tyrosine in Caenorhabditis elegans. Journal of Biological chemistry 294(24):9536-9554

4. Li XJ, Jiang C, Xu N, Li JX, Meng FY, Zhai HQ (2018) Sorting and identification of Rehmannia glutinosa germplasm resources based on EST-SSR, scanning electron microscopy micromorphology, and quantitative taxonomy. Industrial Crops Products 123:303-314

5. Li XX (2007) Cloning of enzymes related to cellulose biosynthesis in soybean and its genetic transformation into potato. Capital Normal University, Beijing, p 05

6. Liu Y, Yang XY, Zhao YH, Yang YS, Liu ZL (2020) An effective method for Agrobacterium tumefaciens-mediated transformation of Jatropha curcas L. using cotyledon explants. Bioengineered 11(1):1146-1158

7. Lu X, Lei H, Wang F, Huang C, Wu SW (2013) Molecular cloning and overexpression of the tyrosine aminotransferase (TAT) gene leads to increased rosmarinic acid yield in Perilla frutescens. Plant Cell Tissue Organ Culture 115(01):69-83

8. O'Connor SE (2015) Engineering of Secondary Metabolism. Annual Review of Genetics 49:71-94

9. Ouyang XF (2017) Determination of catalpol and verbascoside in different varieties of Rehmannia glutinosa by high performance liquid chromatography. Inner Mongolia Traditional Chinese Medicine 36(20):132-133

10. Qi M, Xiong AZ, Li PF, Yang QM, Yang L, Wang ZT (2013) Identification of acteoside and its major metabolites in rat urine byultra-performance liquid chromatography combined with electrospray ionization quadrupole time-of-flight tandem mass spectrometry. J Chromat Ogr B 940:77-85 
11. Saimaru H, Orihara Y (2010) Biosynthesis of acteoside in cultured cells of Olea europaea. Journal of Natural Medicines 64(2):139-145

12. Wang FQ, Zhi JY, Zhang ZY, Wang LN, Suo YF, Xie CX, Li MJ, Zhang B, Du JF, Gu L, Sun HZ (2017) Transcriptome analysis of salicylic acid treatment in Rehmannia glutinosa hairy roots using RNA-seq technique for identification of genes involved in acteoside biosynthesis. Front Plant Sci 8:787

13. Wang HB, Dong QL, Duan DY, Zhao S, Li MJ van, Ma NS, Mao FW K (2018) Comprehensive genomic analysis of the tyrosine aminotransferase (TAT) genes in apple (Malus domestica) allows the identification of MdTAT2 conferring tolerance to drought and osmotic stresses in plants. Plant Physiology Biochemistry 133:81-91

14. Xie J, Tang N, Xia C (2019) A transgenic plant with increased phenylglycoside content and its production method and process: China, Copy number: 17538001[P]. 2019-04-29

15. Xu JJ, Fang X, Li CY, Yang L, Chen XY (2020) General and specialized tyrosine metabolism pathways in plants. aBIOTECH 1:97-105

16. Zhou YQ, Wang XN, Wang WS, Duan HY (2016) De novo transcriptome sequencing-based discovery and expression analyses of verbascoside biosynthesis-associated genes in Rehmannia glutinosa tuberous roots. Mol Breeding 36(10):139

\section{Figures}




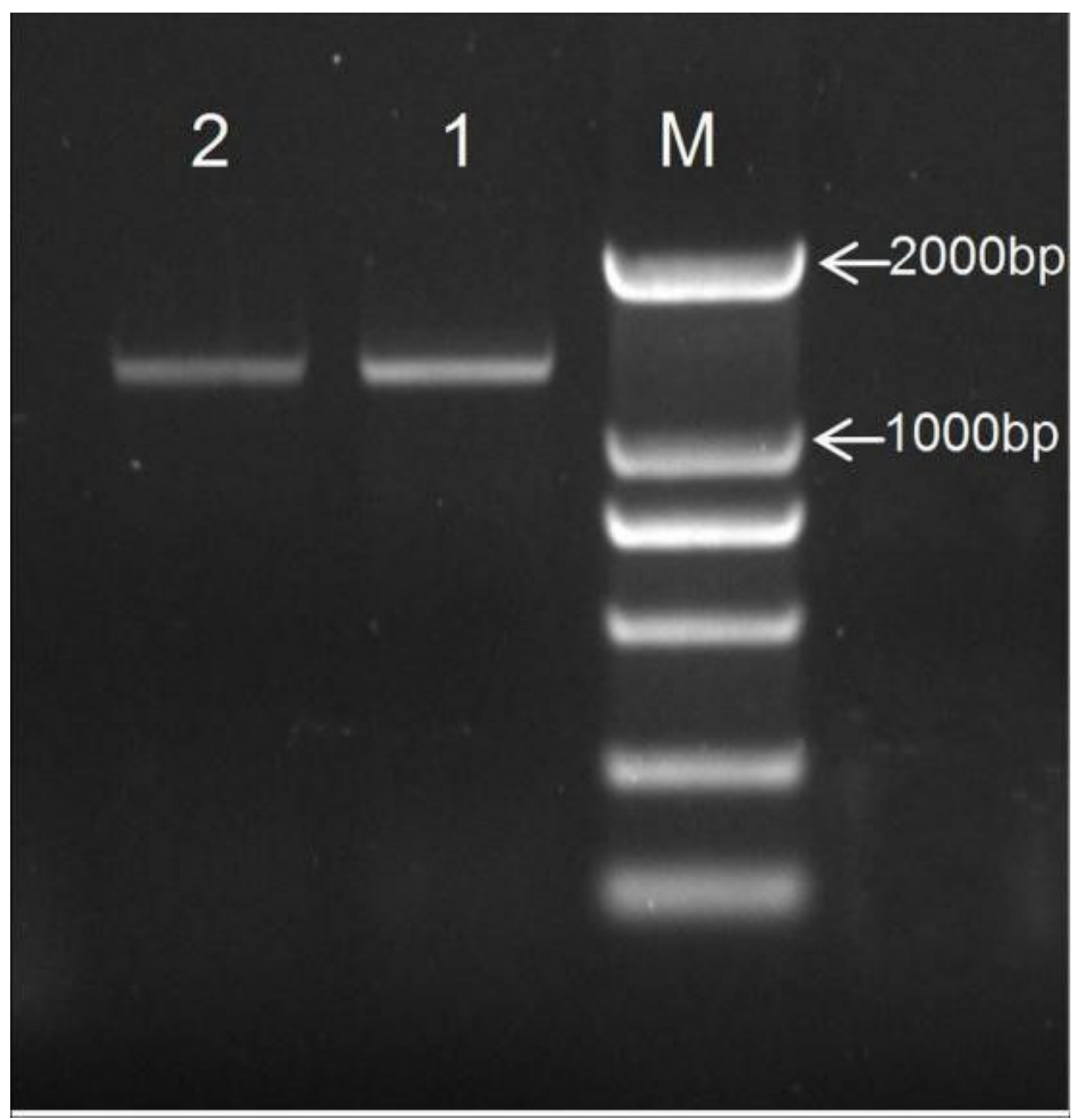

Figure 1

The full-length cDNA (RgTAT) $M \otimes T$ rans2K® DNA marker, 1-2: RgTAT 


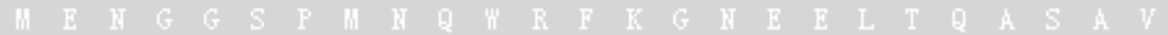
ACCATTAGAGGIGIGCTAAACATECTCATGGAGATCTCAACTAAATGAACOCGACCGITATTCCGITEGCI

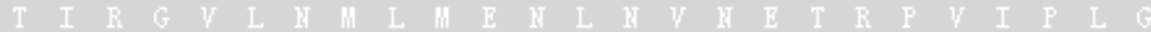

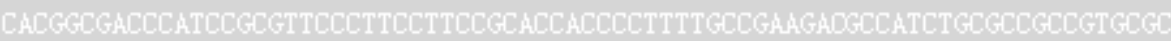
$\begin{array}{lllllllllllllllllllllllll}H & G & D & P & S & A & F & P & S & F & R & T & T & P & F & A & E & D & A & I & C & A & A & V & B\end{array}$

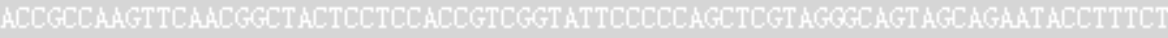

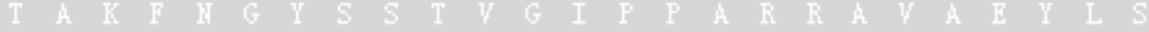
AAGGACCTTCCATACAAGCTATCACCTGATGATGITTCCTGACTATTGGATIACTCAACATTAGAGCCATI $\begin{array}{lllllllllllllllllllllllll}K & \text { I } & \text { L } & \text { P } & \text { I } & \text { K } & \text { L } & \text { S } & \text { P } & \text { I } & \text { I } & V & \text { F } & \text { L } & \text { I } & \text { I } & G & C & \text { I } & \text { Q } & \text { A } & \text { L } & \text { E } & \text { A } & \text { J }\end{array}$ ACCACCGICCTTECTCGCCCCEGIGCTAACATITECTICCACGACCAGCCTITCTTACTACGAGCTAGACCC

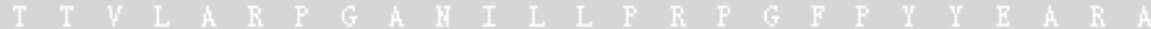

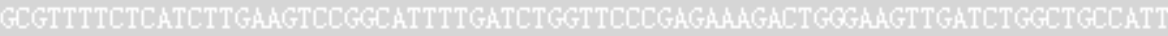

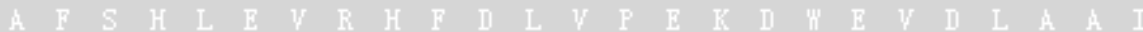

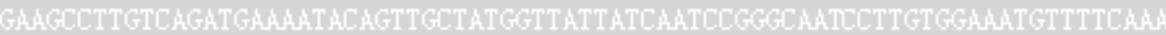

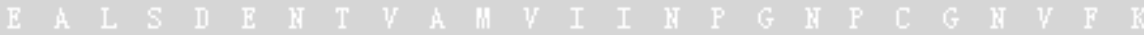
TATGACCATTTGAGAGGTTCAGAGACAGCAAGAACCITGCATTTAGTGTCGCCGATGAGIGTATGAC

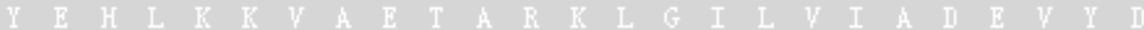

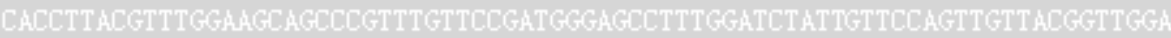

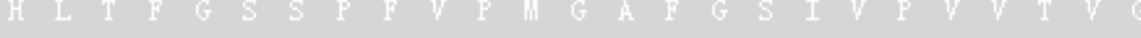
TCAATATCGAGAGATGATTGITCCTGGTTGGAGCTTGECTGECTTGTCACTAATGATCCAGATGGATCCTI $\begin{array}{lllllllllllllllllllllllll}S & I & S & K & R & \| & I & V & P & G & \| & R & L & G & \| & L & V & T & \mathbb{A} & I & P & I & G & I & I\end{array}$

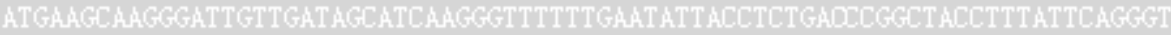

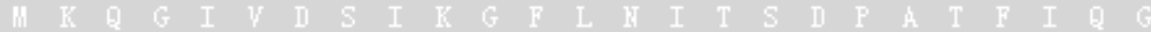
GCAGITCCACAGATCCITGAAATACCCCGACGATITCTITCIGAAATTGIGATACACITAGAGATCTECA

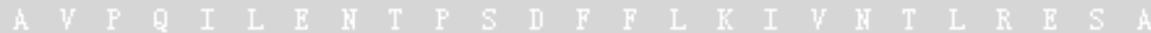
GAGATATECTACATAGACCAAGGAATCCCCTCCATAACTTCCCCACCAHLCCGAGGATCCATGITTGIS

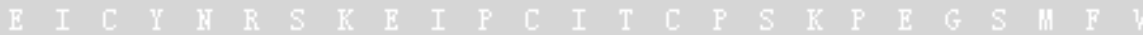

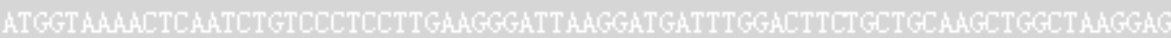
II $\quad$ V

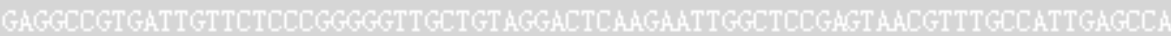

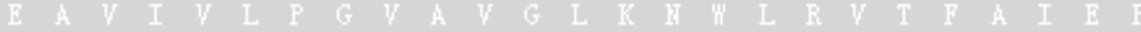
TCATCTCTTGAGATGGICTTGGCCGATAhAGCTTICTATCATAGACACGCTARGAACAATE

\section{Figure 2}

The base sequence of RgTAT and the amino acid sequence of its coding protein *: Stop code. The numbers on the right: the number of the first base (up) or amino acid (down) in every line. 
$\operatorname{Rg}$ TAT

Si_TAT

Eg TAT

$\mathrm{Sb}$ TAT

TC TAT

At_TAT

Consensus

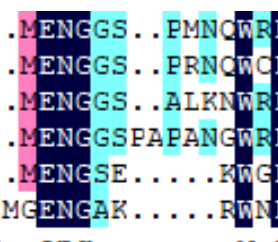

FGNEELTCA SAVTIRGVLMLMENINVNETRPVIPLGHGDPSPFPSFRTTF FRRNEELTRA SAVTVR GVLNMVMEHLNASDFRPVIPLGHGDPSA FPSFRTT L IGNEKLTCA SA ITVRGVLNMLMENINPDEZRPVIPLGHGDPSA FPSERTT PL RGNDDLTQASSITVRGVLNMLMGNINSDDTRPVIPLGHGDPSP FPSFTTFF QGKQEMNAAAA ITVRGVLNMLM GN LNEDDSRPVIPLGHGDPSHFPSHRTSA GANEVVERSNSITIRDYLNTL INOLDGGDVRPVIPLGHGDPSFEPSFRTDQA

rpviplghgdps fpsf $t$

t $r$ ln

Rg_TAT

Si TAT

Eg TAT

$\mathrm{Sb}$ TAT

TC TAT

At_TAT
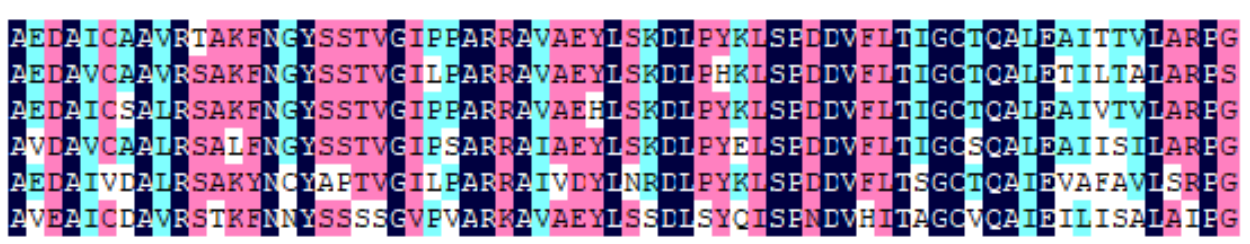

Rg_TAT ANILLPREGEFYEARZAFSHIËRFEDLVEEKLF̈EVDLAAIEZISDENTVAMVIINEGNPCGNVEKY

Si TAT

Eg TAT

Sb_TAT

ANILLPREGFE

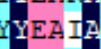

LVP

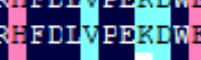

BVDIAAVEZ ISDEN TVAMVIINEGNPCGNVHKY

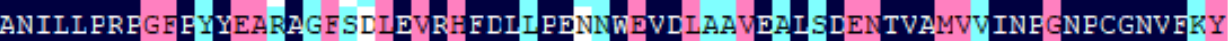

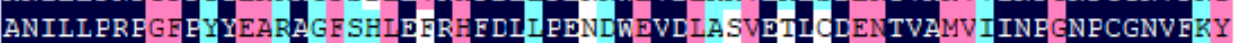

TC_TAT ANILLPREGEFYYASAAYNCFEVRHFDLIPEKGWBVDIDAVETIZDENTVAMVIINEGNPCGNVFSY

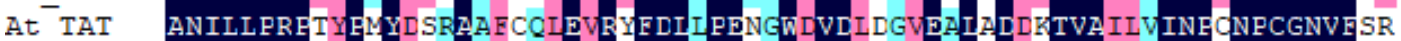

Consensusanillprp py a er fdl pe w vd el d tva inp npcgnvf

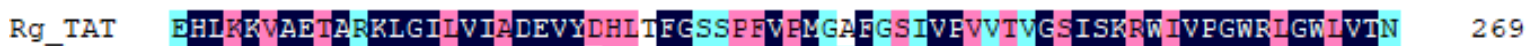

Si_TAT EHLRKVAETRRLGIIVIZDEVYSHITEGGSP FVEMGVGSIVFVVTVGSISKRWIVPGWRIGWIVTN 269

Eg_TAT DHLRKAETRRRLIIVIZDEVYDHITEGESP FVEMGVGSIVEIITVGSISKRWIVPGWRIGWIVTH

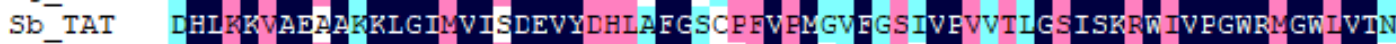

TC_TAT BHLRKVATARKLGIIVIZDEVYDNLA FGSTKYVEMRIFASTVEVITIGSISKKWIVPGWRIGWIVTS

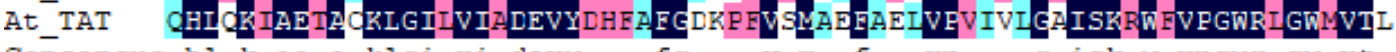

Consensus hl k ae a klgi vi devy fg $\mathrm{v} m$ f vp g isk w vpgwr gw vt

Rg_TAT

Si TAT

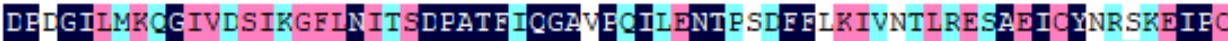

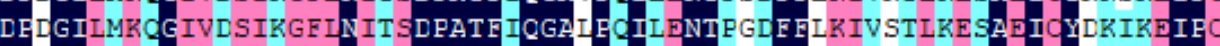

Eg_TAT DENGILMKCGIVDSIKGEINITSDPAT FM QGAVEEILENTPADFETKIVSTLKESA_IOYDRIKBIEC

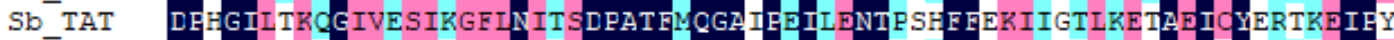

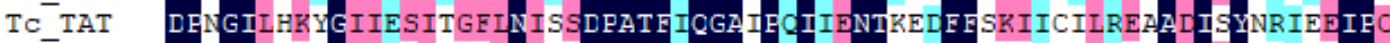

At_TAT DEHGIMKDSGFVQTIINVWNMSTDPAT FIQGAMEIII INTKEEF SSKLEMVKKOZI IOYEELMKIFC

Consensusdp gi g

g $n$ dpatf gga pi nt ff

a $i \mathrm{y}$

ip

201

201

201

203

198

199

269

271

266

267

337

337

337

339

334

335

Rg_TAT ITCESKPEGSMFVMVKLNLSIIEGIKDLLDFCOKLAKEEAVIVLPGVAVGIKN R̈IRVTFRIEPSSIDD

Si_TAT ITCESKPEGSMFVMVKLNLSILEDIKDDTDFCOKLFTEESVIILPGVAVGSKYKILVTERIEPSSIDD

$\mathrm{Eg}^{-}$TAT ITCESRPEGSMFVMVKLNLSIIEGFMDDTDFCOKLAKEESVIVLPGVAVGLKN RIRITFAIEPSSIDD

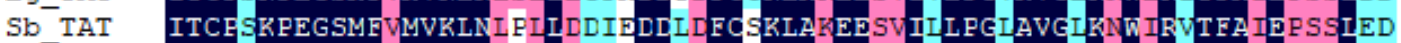

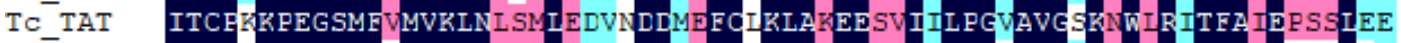

At_TAT ITCEOKPEGSMETMVKLNFSILEDISDDLIFCSKLAREESM IILPGQAVGIKNWIRITEAVELEIIIE

Consensusitcp kpegsmf mvkln 1 dd fc kla ee i lpgavg $k$ w tfa e 1

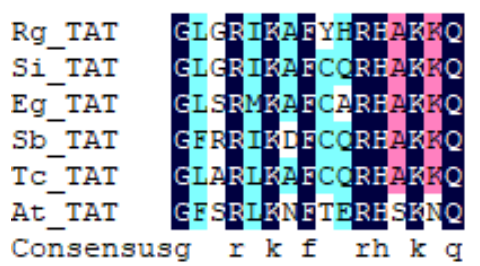

405

405

405

407

402

403

421

421

421

423

418

419

\section{Figure 3}

A multiple amino acid sequence alignment of RgTAT protein to the TATs from other plant species using a

Blast search within the GenBank database. Rg: Rehmannia glutinosa. Si: Sesamum indicum. Eg:

Erythranthe guttatus. Sb: Scutellaria baicalensis. Tc: Theobroma cacao. At: Arabidopsis thaliana 


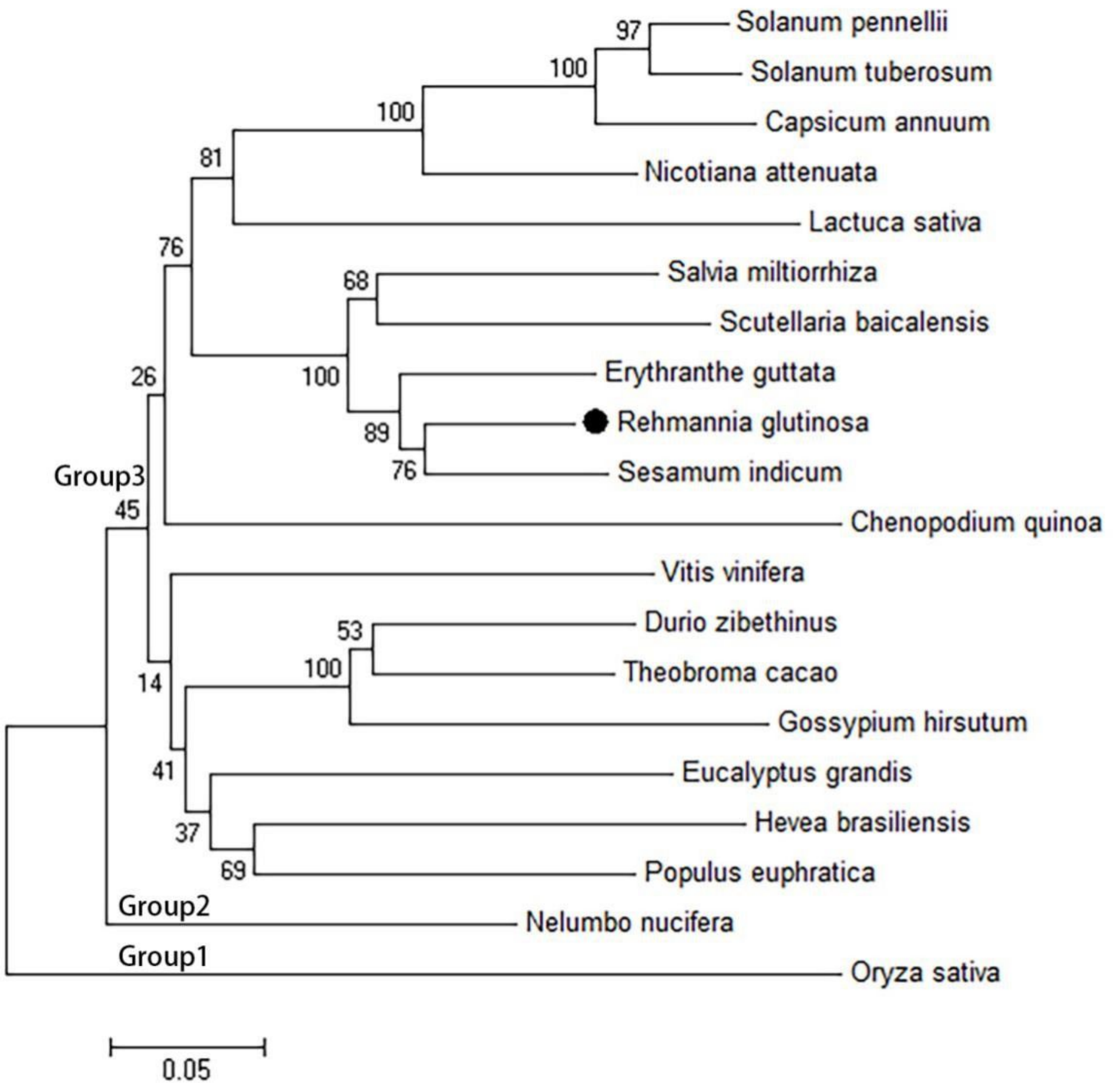

Figure 4

Phylogenic tree analyses of RgTAT and the TATs from other plant species using Neighbor Joining $(\mathrm{NJ})$ method. @: Rehmannia glutinosa RgTAT. Solanum pennellii (Accession number: NP_001310376.1), Solanum tuberosum (Accession number: XP_015161160.1), Capsicum annuum (Accession number: XP_016580784.1), Nicotiana attenuate (Accession number: XP_019247924.1), Lactuca sativa (Accession number: XP_023758259.1), Salvia miltiorrhiza (Accession number: AGW27212.1), Scutellaria baicalensis (Accession number: AIV98133.1), Erythranthe guttatus (Accession number: XP_012831711.1), Sesamum indicum (Accession number: XP_011100354.1), Chenopodium quinoa (Accession number: 
XP_021733335.1), Vitis vinifera (Accession number: XP_002276551.1), Durio zibethinus (Accession number: XP_022748926.1), Theobroma cacao (Accession number: XP_017971878.1), Gossypium hirsutum (Accession number: XP_016692235.1), Eucalyptus grandis (Accession number: XP_010052168.2), Hevea brasiliensis (Accession number: XP_021641638.1), Populus euphratica (Accession number: XP_011029070.1), Nelumbo nucifera (Accession number: XP_010250299.1), Oryza sativa (Accession number: XP_015623362.1)

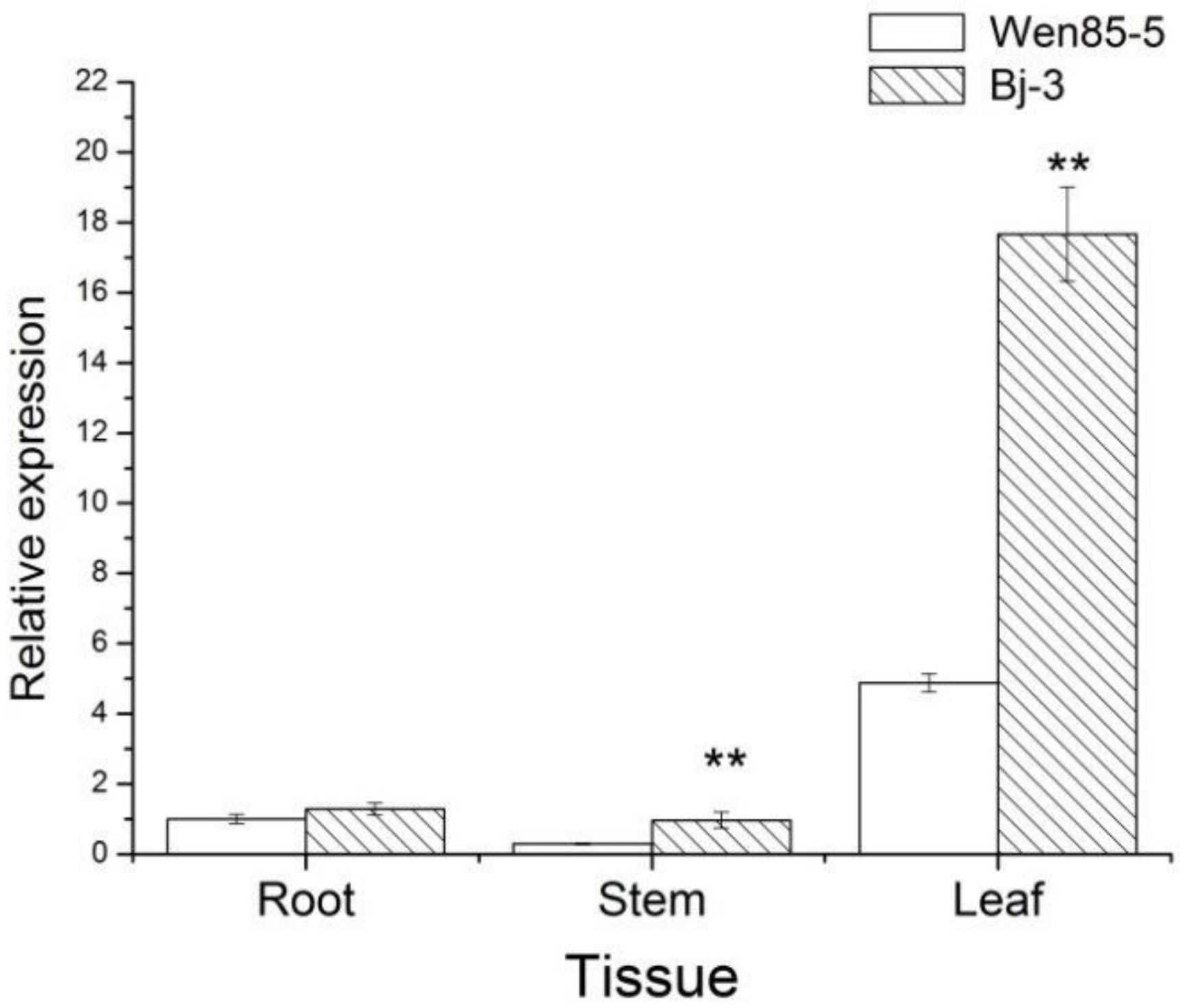

\section{Figure 5}

The relative expression of RgTAT in the different tissues of Rehmannia glutinosa cultivars Wen85-5 and

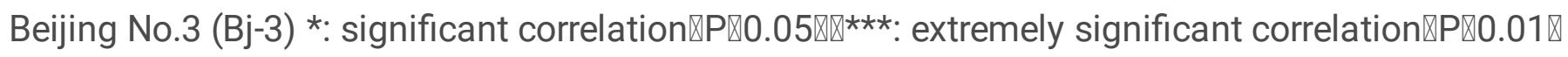




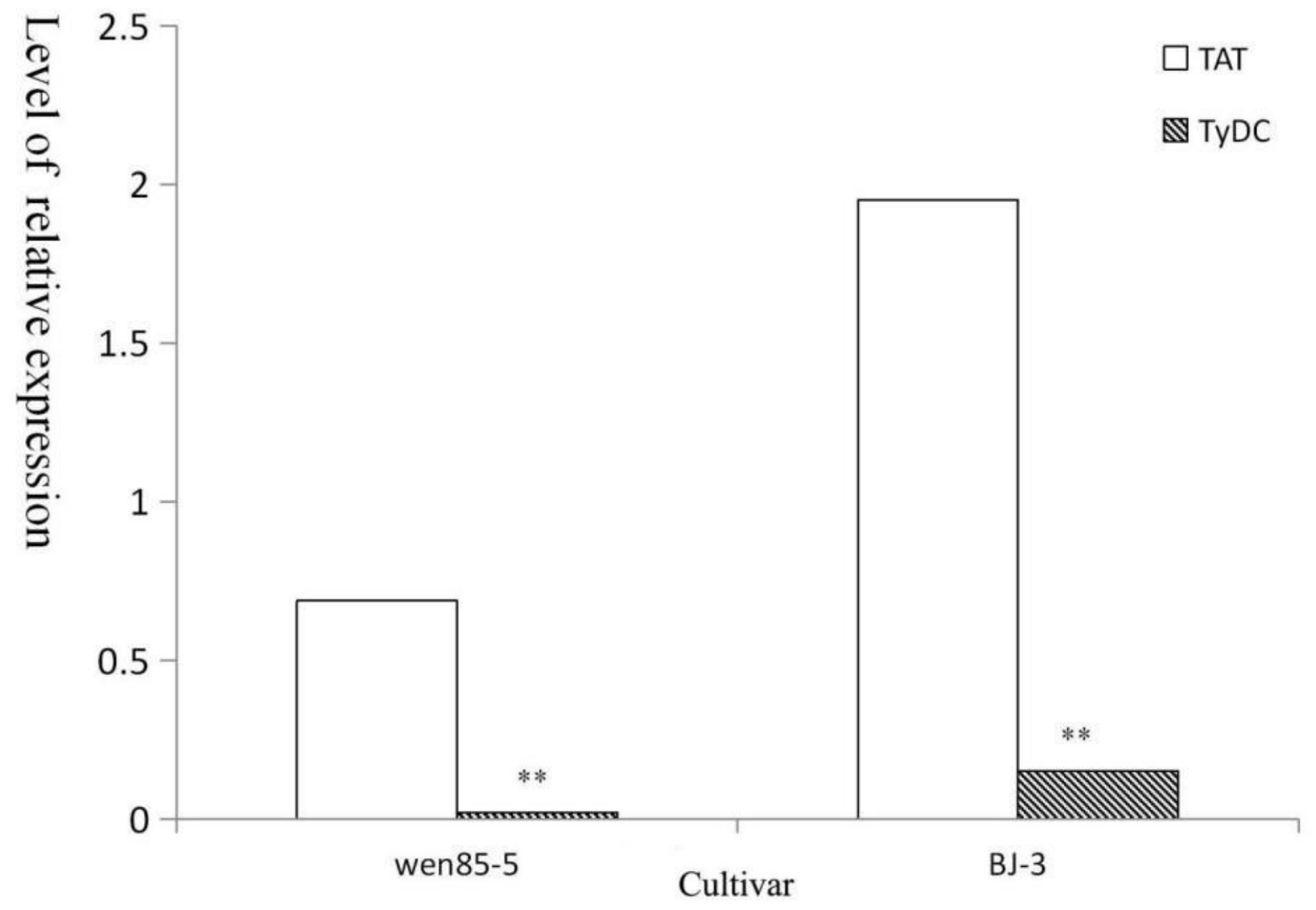

Figure 6

The correlation of RgTAT gene expression level to RgTyDC gene expression level in cultivars Wen85-5 and

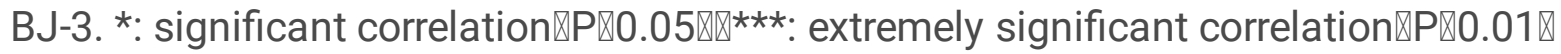




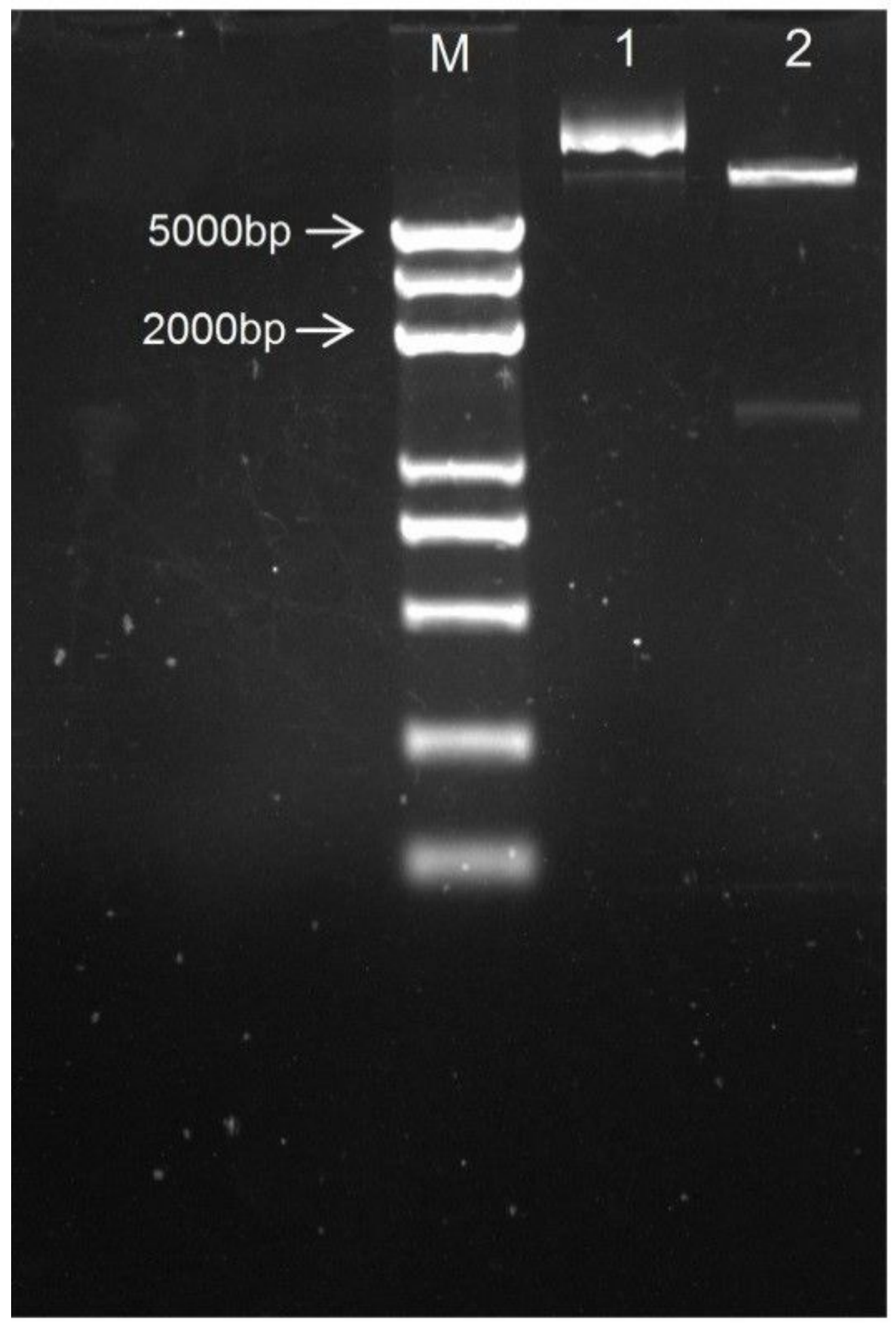

\section{Figure 7}

The verification of the recombinant plasmid pCAMBIAL1300-35S-RgTAT by double digestion M $\triangle D N A$ Marker. 1:The recombinant plasmid pCAMBIAL1300-35S-RgTAT. 2: The products of the recombinant plasmid pCAMBIAL1300-35S-RgTAT by double digestion. Up: plasmid pCAMBIAL1300-35S. Down: RgTAT. Arrow: RgTAT. 


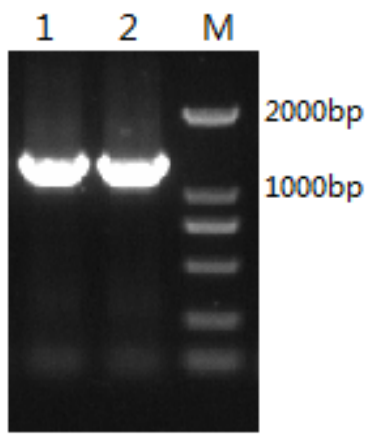

Figure 8

Agarose gel electrophoresis of PCR product from the Agrobacterium transformants harboring RgTAT M】 DNA Marker.1-2هRgTAT 


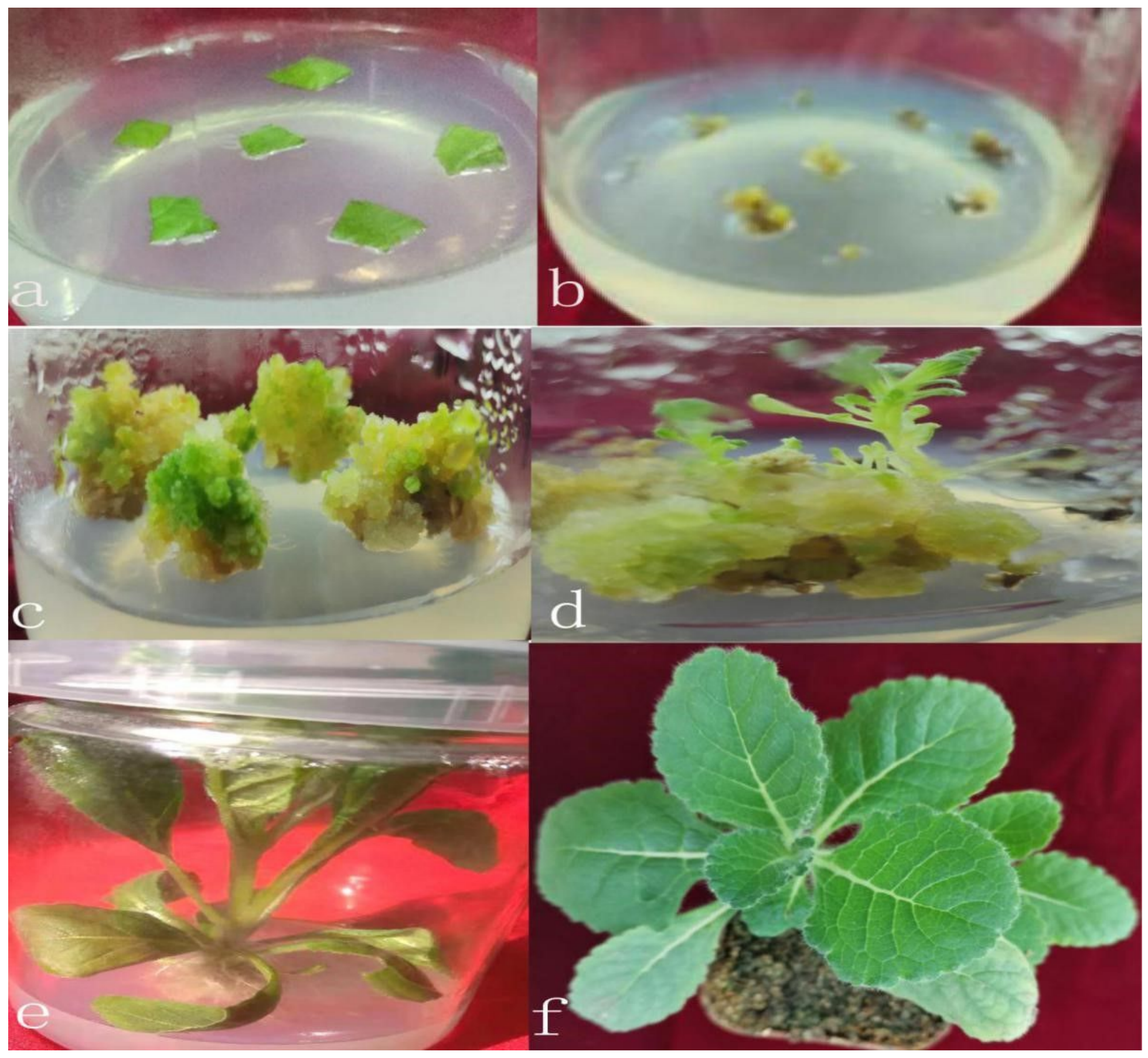

\section{Figure 9}

Transformation figures a. The pieces of leaves. b. calli. c. propagated calli. d. differentiated shoots from the calli. e. regenerated plantlet. f. transplanted plantlet in a flowerpot. 


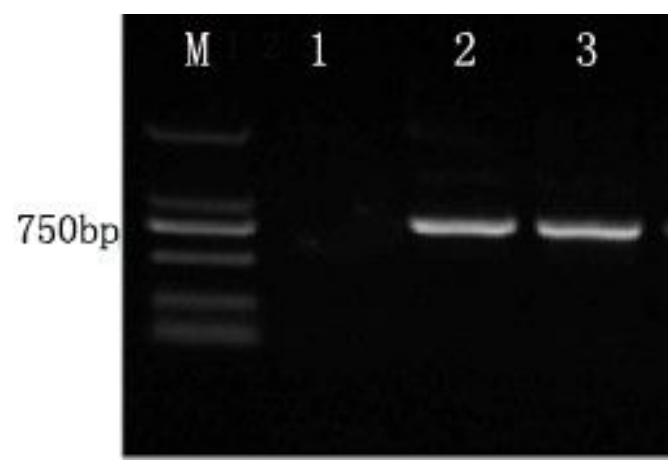

Figure 10

Agarose gel electrophoresis of hygromycin resistance genes amplified by PCR from T0 transformants. Lane M『DNA marker. Lane1区Non-transformant. Lanes2-3凶No.1 and No.2 of T0 transformants. 